\title{
ALGORITMO PARA LA ESTIMACIÓN DEL ÁNGULO ANTEROPOSTERIOR DE UNA MULETA SENSORIZADA
}

\author{
Iñigo Sesar, Asier Zubizarreta, Itziar Cabanes \\ Departamento de Ingeniería de Sistemas y Automática, Universidad del País Vasco (UPV/EHU) \\ Facultad de Ingeniería de Bilbao
}

\begin{abstract}
Resumen
En los procesos de rehabilitación del miembro inferior, la monitorización de la marcha es una fuente sustancial de información para el fisioterapeuta. Sin embargo, muchos de los dispositivos propuestos en la literatura se basan en sistemas invasivos para el paciente. La integración de sensores en los dispositivos de ayuda técnica, como muletas, permite solventar este problema. Así, en este trabajo se propone el uso de un algoritmo que permite estimar la inclinación anteroposterior de la muleta, basado en el uso de un sensor de fuerza, un inclinómetro y un giróscopo. La inclinación anteroposterior puede servir para definir indicadores que se correlacionen con escalas clínicas empleadas para evaluar el estado funcional del paciente y su actividad física.
\end{abstract}

Palabras clave: Muleta sensorizada, neurorehabilitación, monitorización de la marcha.

\section{Introducción}

La salud es una de las áreas con mayor actividad investigadora en los últimos años. Los avances tecnológicos han posibilitado nuevos sistemas de diagnóstico y tratamiento que tienen un impacto directo en nuestra calidad de vida. Sin embargo, entre las diferentes áreas relacionadas con la salud, la rehabilitación es una de las que menos inserción tecnológica presenta. Tradicionalmente, la evaluación del paciente se ha hecho usando como estándares test clínicos con un carácter subjetivo y escalas con poca granularidad $[1,2]$.

La evaluación objetiva y precisa del paciente es un aspecto clave en la rehabilitación, especialmente en pacientes que requieren una rehabilitación del miembro inferior, dado el impacto que tiene en su calidad de vida [3]. En la literatura, la monitorización de la marcha se realiza principalmente usando tres tipos de dispositivos: plataformas de presión, sistemas de captura de movimiento (MCS) o sensores vestibles. La primera alternativa es fácil de usar y precisa, pero no proporciona información sobre el movimiento de los miembros del cuerpo y presenta rango limitado de uso [4]. El uso de sistemas de captura de movimiento proporciona una captura integral del cuerpo con gran precisión, aunque su puesta en marcha es costosa y el rango de visión es limitado [5]. Por último, el uso de sensores vestibles es mínimamente invasivo y no está limitado en rango, aunque la calibración de los mismos, la unión al paciente y el post-procesamiento presentan retos a tener en cuenta [6].

Para evitar los problemas derivados de los anteriores elementos de monitorización, algunos autores han propuesto el incorporar sensores en los dispositivos pasivos usados habitualmente por pacientes, como, por ejemplo, las muletas $[2,7,8]$. Esta alternativa, no es invasiva para el paciente y no está limitada en rango, aunque requiere de procesar adecuadamente los sensores integrados para correlacionar el movimiento de la muleta con la actividad del paciente. Estudios recientes [9] sugieren que el uso de muletas sensorizadas puede influir positivamente en la recuperación del paciente, dado que permiten evaluar al mismo tanto dentro como fuera de la clínica, corregir el mal uso de las mismas, o evaluar la cantidad de actividad física que realiza el paciente para personalizar la terapia de rehabilitación.

A tal fin, es fundamental una adecuada selección de sensores, que permita extraer la mayor cantidad posible de datos de la marcha. El uso de sensores de medida inerciales (IMUs) es habitual en estos dispositivos. Sin embargo, éstos presentan un problema de deriva que puede provocar errores importantes al procesar la señal [6,7]. Por ello, en este trabajo se propone un algoritmo que fusiona los datos que ofrece un giróscopo, un inclinómetro y un sensor de fuerza, con el fin de extraer una estimación del movimiento anteroposterior de la muleta, y con ello caracterizar el movimiento del paciente.

El resto del artículo está estructurado de la siguiente forma. En la sección 2, se presenta una descripción breve de la muleta sensorizada, en la sección 3 se presenta el algoritmo de estimación de la inclinación anteroposterior de la muleta, en la sección 4, se valida el algoritmo usando un sis- 
tema de captura de movimiento. Por último, las ideas más importantes son resumidas en las conclusiones.

\section{Muleta Sensorizada}

El prototipo de muleta sensorizada se muestra en la Figura 1. El sistema cuenta con una contera adaptable fabricada en aluminio que incorpora un alojamiento para los sensores y la circuitería de acondicionamiento. Adicionalmente, la estructura de aluminio consta con un vástago que se conecta a la punta de goma de la muleta, permitiendo la transmisión de fuerzas desde ésta hasta el sensor de fuerza alojado en su interior.

La estructura de la contera aloja tres tipos de sensores. Por un lado, las fuerzas axiales de la muleta son medidas con un sensor HBM C9C de $1 \mathrm{kN}$ de fuerza nominal. La salida de este sensor es amplificada por un circuito INA118 para generar una tensión analógica que pueda ser medible por el dispositivo de captura. Los ángulos de inclinación anteroposterior y lateromedial de la muleta son medidos por un inclinómetro de dos ejes SCA100T-D02, mientras que la aceleración y velocidad angular son medidos por una IMU MPU-6000.
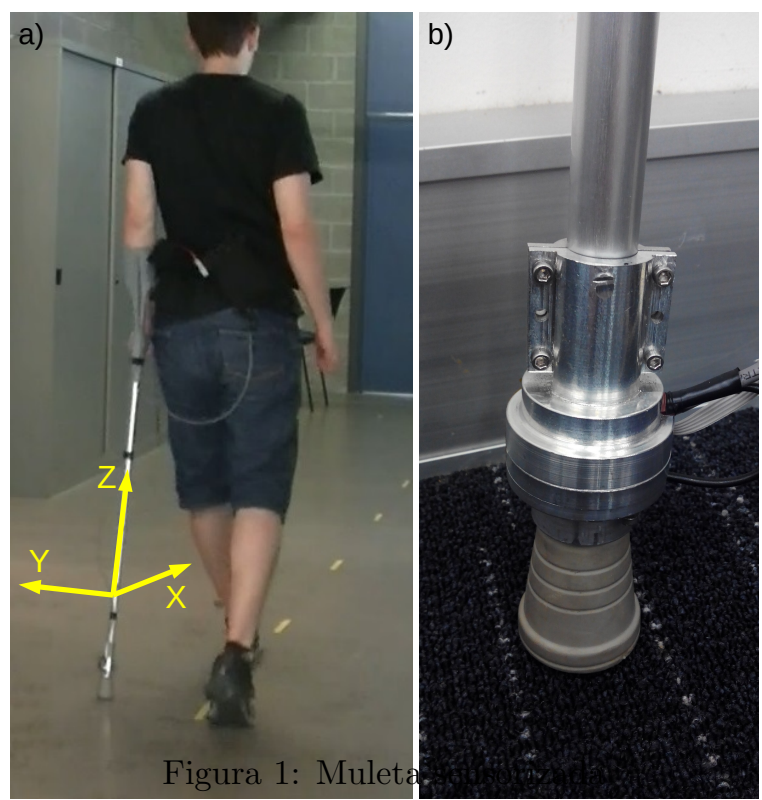

El sistema de captura y procesado se ha diseñado usando una myRIO de National Instruments, que se programa en Labview RT. El sistema de adquisición diseñado captura con una frecuencia de $100 \mathrm{~Hz}$ los datos de los sensores. Los datos son guardados en archivos LVM que posteriormente pueden ser descargados para procesarlos.

Nótese que si bien la muleta incorpora un inclinómetro, la medida de éste sólo es correcta en situaciones de bajas aceleraciones. En caso de impactos, como el de la muleta contra el suelo, las medidas del inclinómetro no son fiables. Tampoco son fiables estas medidas en las fases de la marcha en las que la muleta se mueve sin contacto con el suelo. De igual modo, los datos que proporciona la IMU presentan una deriva con el tiempo, que puede ser importante en el caso de dispositivos de bajo coste como el integrado en el dispositivo. Es por ello que se requieren de algoritmos adecuados para obtener indicadores para monitorizar la marcha.

\section{Algoritmo de estimación}

La estimación de la inclinación antero-posterior de la muleta se puede emplear para estimar otros parámetros de la marcha (longitud de paso, distancia recorrida, etc.) que podrían servir para cuantificar objetivamente el estado funcional del paciente $\mathrm{y}$, por tanto, adaptar las terapias al mismo. Sin embargo, tal y como se ha analizado en la anterior sección, ninguno de los sensores incorporados en la muleta, por si solo, permite una estimación fiable de esta variable, dado que el giróscopo sufre de una deriva a lo largo del tiempo y la medida del inclinómetro es perturbada por las aceleraciones durante la marcha. Por ello, se

c) requiere definir un algoritmo de estimación que, usando los datos de todos ellos, ofrezca esta variable.

Con el fin de calcular una estimación de la inclinacióngnteroposterior, en esta sección se propone un algoritmo que combina los datos que ofrece el serisor de fherza, el inclinómetro y el giróscopo. Con el fim de explicar el algoritmo hay que hacer referencia a las dos fases de movimiento de la muletaf a) apoyo, cuando la muleta impacta contra el suelo y se usa para impulsarse, y b) oscilación, cuando la muleta avanza sin contacto con el suplor Hay-que tener en cuenta que las fases de moyimiento de la muleta no se corresponden exactamente con las fases de la marcha del usuario. Nótese que al micio de la fase de apoyo, la muleta impacta eontra el suelo, con lo que la medida del inclinómetro no ofrece buena señal. Sin embargo, tras este fansitorio, la muleta está pivotando respecto de un punto fijo, con lo que su inclinación puede ser medida por el inclinómetro. La identificación de estas fases se puede realizar de forma sencilla usando la medida del sensor de fuerza, que indica si existe contacto con el suelo o no.

Por tanto, la idea principal del algoritmo es la de integrar los datos que ofrece el giróscopo para estimar el ángulo asociado a la inclinación, pero corrigiendo la deriva con la medida del inclinómetro en un punto determinado de la fase de apoyo. Para 


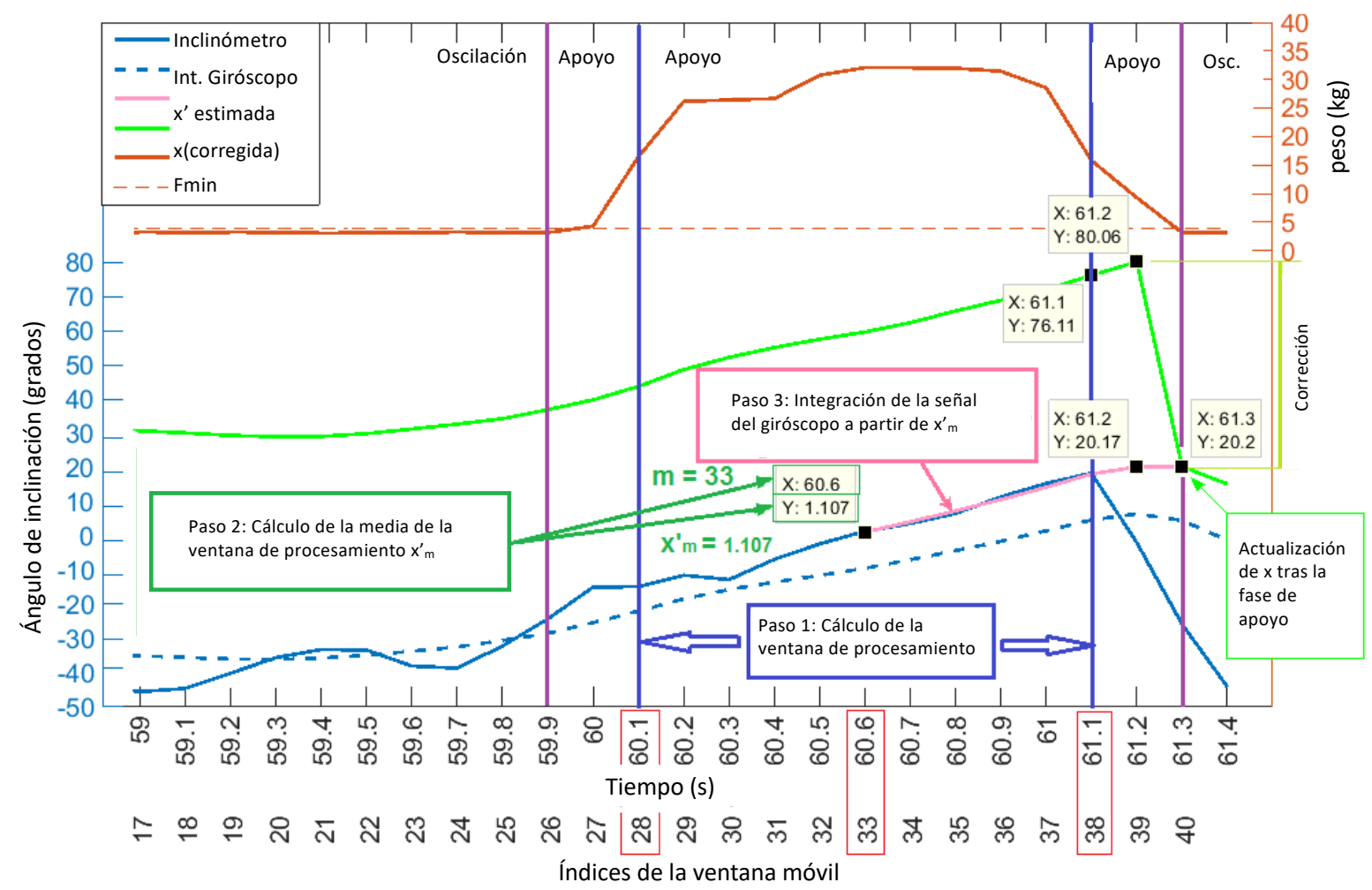

Figura 2: Interpretación del algoritmo

ello, se establece una ventana de tiempo móvil, que abarca el último ciclo de movimiento de la muleta (oscilación + fase de apoyo), que se procesa y se usa para corregir la deriva al final de cada ciclo.

La Figura 2 resume las etapas principales del algoritmo (nótese que los datos están exagerados para explicar el procedimiento). En operación normal, el ángulo anteroposterior es estimado mediante una integración discreta de la velocidad angular ofrecida por el giróscopo. Así, para $t=k$

$$
x_{k}=x_{k-1}+\frac{T_{s}}{2}\left(u_{k}+u_{k-1}\right)
$$

donde $x_{k}$ es el nuevo valor de la inclinación estimada, $x_{k-1}$ es el valor estimado anterior, $T_{s}$ es el periodo de muestreo, y $u_{k}, u_{k-1}$ son los dos últimos valores medidos por el giróscopo, después de ser filtrados con un filtro paso bajo de $10 \mathrm{~Hz}$.

En cada instante de tiempo, se analizan los datos del giróscopo, el inclinómetro y el sensor de fuerza, que han sido almacenados en la ventana que abarca el último ciclo de movimiento. El primer paso es el de detectar el comienzo y final de la fase de apoyo, que puede realizarse simplemente definiendo un valor mínimo de fuerza de contacto $F_{\text {min }}$, a partir del cual se supone que la muleta está en contacto con el suelo. Una vez detectada la ventana asociada a la fase de apoyo, se han de eliminar las muestras iniciales y finales, dado que pueden existir transitorios asociados a las perturbaciones causadas por el impacto de la muleta y posterior levantamiento de la misma. Una vez identificado el tramo central de la ventana, se calcula el valor medio de los valores del inclinómetro en este intervalo $x_{m}^{\prime}$. Tomando este valor como origen, se procede a integrar los valores medidos por el giróscopo hasta el final de la ventana, con el fin de estimar el valor que debería tener la inclinación en el instante actual $x_{k}^{\prime}$,

$x_{t}^{\prime}=x_{t-1}^{\prime}+\frac{T_{s}}{3}\left(u_{t-1}+u_{t}+u_{t+1}\right) \quad t=m+1, \ldots k$

donde $m$ es el índice asociado a $x_{m}^{\prime}$, y el algoritmo se ejecuta de $t=m+1, \ldots k$. De este modo, es posible calcular un valor estimado de la inclinación $x_{k}^{\prime}$. Si ha existido deriva, $x_{k}^{\prime} \neq x_{k}$, por lo que se tomará $x_{k}^{\prime}$ como el valor asociado a $x_{k}$ y se seguirá ejecutando el algoritmo a partir de este punto.

Nótese que el algoritmo sólo actualiza el valor de la medición al final de cada ciclo, usando siempre los datos del ciclo anterior. Esto implica que si la deriva durante un ciclo es pequeña, como suele ser el caso, será posible garantizar una precisión adecuada.

Adicionalmente es reseñable tener en cuenta que 
este algoritmo considera que, durante la fase de apoyo, las aceleraciones y vibraciones a las que está sometido el inclinómetro son pequeñas. De igual modo, es importante tener en cuenta que la muleta, al ser usada, presenta un giro en su eje $\mathrm{z}$, con lo que realmente el ángulo estimado no es exactamente la inclinación anteroposterior, sino el giro en torno al eje $y$ local de la muleta (Fig. 1). La inclinación exacta requeriría la estimación de este ángulo $z$, aunque a efectos prácticos, es posible usar este giro como aproximación de la inclinación anteroposterior, con el fin de derivar posteriormente indicadores que cuantifiquen el estado del paciente.

\section{$4 \quad$ Validación}

Con el fin de validar el algoritmo en un entorno dinámico, se ha realizado un ensayo con dos voluntarios de $168 \mathrm{~cm}$ y $182 \mathrm{~cm}$ de alto y 70 y 68 $\mathrm{kg}$ de peso respectivamente. Las pruebas se han realizado en una sala equipada con un sistema $\mathrm{Vi}$ con de captura de movimiento 3D, lo que ha limitado el diseño de la prueba. De este modo, se ha definido una trayectoria basada en andar en línea recta $5 \mathrm{~m}$, dar media vuelta y volver al punto de origen, repitiendo 2 veces este ciclo y completando un total de $20 \mathrm{~m}$ de distancia. Se les ha pedido a los voluntarios que caminen a un paso normal durante el trayecto, intentando apoyar el máximo peso sobre la muleta. Los ensayos se realizaron con la aprobación del Comité de Ética de la UPV/EHU (código M10/2016/295MR1).

El movimiento de la muleta ha sido capturado con el sistema Vicon usando una serie de marcadores de referencia, a través de los cuales es posible determinar en cada instante cuál es la posición y orientación de la muleta respecto al Sistema de Referencia Global. Con el fin de garantizar la sincronización de los datos capturados por el Vicon y la myRIO de la muleta, ambos se han configurado con un periodo de adquisición de $100 \mathrm{~Hz}$, y al inicio de cada ensayo, se ha dado un golpe con la muleta para establecer un punto de sincronización.

Para estos ensayos, se ha establecido una ventana de tiempo deslizante de 4 segundos en el sistema de captura de la muleta, de modo que se pueda detectar el intervalo correspondiente a la fase de apoyo. La detección del inicio y fin del apoyo se ha realizado usando un valor de fuerza mínimo $F_{\text {min }}$ equivalente a $3.82 \mathrm{~kg}$. Tal y como se ha detallado en la sección anterior, a la hora de procesar la última fase de apoyo de la ventana de tiempo, se han descartado las muestras de los primeros y finales $200 \mathrm{~ms}$ del intervalo para eliminar los efectos dinámicos del golpe de la muleta contra el suelo.
Los resultados para el voluntario 2 se muestran en la Figura 3. En ella se muestra la evolución temporal de las señales adquiridas por los sensores de la muleta (integral de la señal del giróscopo, inclinómetro y sensor de fuerza), la señal corregida estimada por el algoritmo propuesto y el ángulo medido a partir de los datos proporcionados por el sistema de captura Vicon, que se tomará como referencia. Como se puede observar, la señal ofrecida por el algoritmo propuesto se asemeja sustancialmente a la del Vicon.

Es importante reseñar que el algoritmo propuesto se actualiza al final de cada fase de apoyo. Esto implica que si existe una deriva o perturbación en la integral del giróscopo, esta sólo podrá ser compensada cuando acabe el ciclo, tal y como se ve en el ciclo 12. En este caso concreto, este ciclo corresponde a cuando el voluntario da media vuelta, con lo que el algoritmo tiene que volver a adaptar la corrección a la nueva dirección. Es importante reseñar, adicionalmente, que al dar media vuelta, la muleta suele tener una ligera inclinación lateromedial, por lo que la componente del giróscopo en el eje $y$ local de la muleta, correspondiente al giro alrededor del eje perpendicular al suelo, no es despreciable e introduce un offset importante, el cual se corrige al final de la fase de apoyo.

Adicionalmente, se observa que la señal corregida es ligeramente superior a la de referencia. Esto es debido al ruido en la medida del inclinómetro y las pequeñas aceleraciones que existen en la fase de apoyo, que han sido despreciadas en el algoritmo. Aun así, el efecto de estas es pequeño y las aproximaciones encajan.

Por último, la Tabla 1 resume los errores RMS por cada ciclo de la muleta cuando se considera únicamente la señal integrada del giróscopo y cuando se considera la corrección propuesta por el algoritmo. Como se puede observar, el algoritmo reduce significativamente el error de estimación en todos los casos, incluso en cambios de dirección. De hecho, si los cambios de dirección bruscos no son considerados, lo que es plausible en un entorno real, el error RMS disminuye a 1.77 grados para el primer voluntario y 2.73 grados para el segundo.

Por tanto, estos resultados demuestran que el algoritmo propuesto presenta potencial para solventar los problemas derivados de la estimación del ángulo anteroposterior de la muleta.

\section{Conclusiones}

La monitorización de aquellos pacientes que usan dispositivos de ayuda técnica como muletas en su rehabilitación, resulta fundamental para personalizar las terapias a los mismos. El uso de muletas 


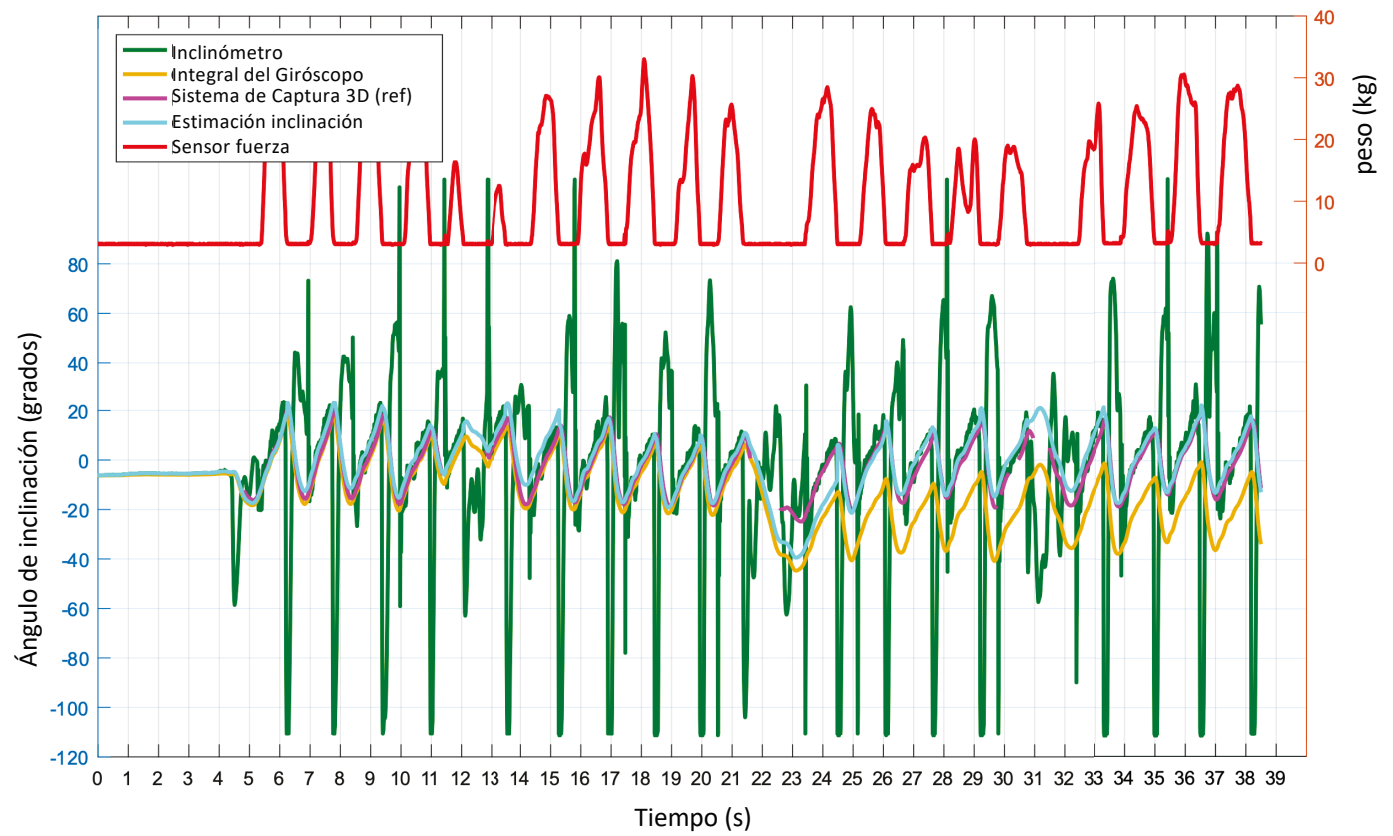

Figura 3: Resultados experimentales del algoritmo

sensorizadas permite proporcionar datos sobre la actividad del paciente que pueden ser útiles. Sin embargo, los sensores inerciales tradicionalmente usados para estos fines presentan derivas y perturbaciones que han de ser subsanadas con el fin de garantizar una buena medición.

En este trabajo se propone el uso de un algoritmo simple de fusión de sensores que, basándose en el comportamiento cíclico de la muleta, hace uso de un giróscopo, un inclinómetro y un sensor de fuerza para ofrecer una estimación precisa del ángulo anteroposterior. El algoritmo propuesto, es fácilmente implementable con sensores de bajo coste y permite un procesamiento rápido y sencillo.

El algoritmo es validado con dos sujetos usando un sistema de captura de movimiento $3 \mathrm{D}$, demostrando que es capaz de reducir la deriva de la señal de los giróscopos y ofrecer un error de estimación RMS de menos de 3 grados.

\section{Agradecimientos}

Este trabajo se ha realizado con la financiación del proyecto DPI2017-82694-R del MINECOFEDER, el Gobierno Vasco mediante la beca PRE_2016_2_0236 y el proyecto del Gobierno Vasco GIU18/162.

\section{English summary}

\section{ESTIMATION OF THE ANTERO- POSTERIOR ANGLE OF A SEN- SORIZED CRUTCH}

\section{Abstract}

In lower limb rehabilitation therapies, gait monitoring is an interesting source of information for the therapist, as it allows to personalise the therapy to the particular needs of each patient. However, most devices proposed in the literature are based on invasive solutions that hamper the motion of the patient. Integrating sensors in assistive devices such as crutches allow to solve this issue. This way, in this work a novel algorithm is proposed, which allows to estimate the anteroposterior motion of the crutch, using a force sensor, an inclinometer and a gyroscope. This estimation can be used to define indicators that can be used to evaluate patient status, being an important source of information for the therapist.

Keywords: Sensorized Crutch, Neurorehabilitation, gait monitoring.

\section{Referencias}

[1] Nordin, N.; Xie, S.Q.; Wünsche, B. Assessment of movement quality in robot-assisted upper limb rehabilitation after stroke: a 
Tabla 1: Errores RMS en grados para los dos voluntarios

\begin{tabular}{cccccc}
\hline Ciclo Nr. & $\begin{array}{c}\text { Voluntario } \mathbf{1} \\
\text { Giróscopo }\end{array}$ & $\begin{array}{c}\text { Voluntario } \mathbf{1} \\
\text { Señal corregida }\end{array}$ & Ciclo Nr. & $\begin{array}{c}\text { Voluntario 2 } \\
\text { Giróscopo }\end{array}$ & $\begin{array}{c}\text { Voluntario 2 } \\
\text { Señal corregida }\end{array}$ \\
\hline $\mathbf{1}$ & 1.16 & 0.99 & $\mathbf{1}$ & 2.58 & 1.52 \\
$\mathbf{2}$ & 2.17 & 0.44 & $\mathbf{2}$ & 2.88 & 3.50 \\
$\mathbf{3}$ & 2.86 & 0.68 & $\mathbf{3}$ & 2.90 & 3.92 \\
$\mathbf{5}$ & 5.88 & 2.91 & $\mathbf{4}$ & 2.84 & 3.10 \\
$\mathbf{6}$ & 7.87 & 2.23 & $\mathbf{6}$ & 3.37 & 5.40 \\
$\mathbf{7}$ & 9.35 & 1.23 & $\mathbf{7}$ & 3.20 & 1.90 \\
$\mathbf{8}$ & 10.01 & 0.58 & $\mathbf{8}$ & 3.70 & 1.90 \\
$\mathbf{1 0}$ & 27.52 & 13.09 & $\mathbf{9}$ & 3.43 & 1.26 \\
$\mathbf{1 1}$ & 29.62 & 1.42 & $\mathbf{1 0}$ & 3.68 & 1.49 \\
$\mathbf{1 2}$ & 32.72 & 2.66 & $\mathbf{1 2}$ & 19.51 & 3.99 \\
$\mathbf{1 3}$ & 32.98 & 2.88 & $\mathbf{1 3}$ & 21.14 & 3.20 \\
$\mathbf{1 5}$ & 27.33 & 10.17 & $\mathbf{1 4}$ & 19.94 & 5.31 \\
$\mathbf{1 6}$ & 26.66 & 3.67 & $\mathbf{1 5}$ & 20.66 & 2.08 \\
$\mathbf{1 7}$ & 28.11 & 2.67 & $\mathbf{1 7}$ & 18.17 & 1.38 \\
& & & $\mathbf{1 8}$ & 18.98 & 3.54 \\
\hline
\end{tabular}

review. Journal of Neuroengineering and Rehabilitation 2014, 11(137), 1-23, DOI. 10.1186/1743-0003-11-137

[2] Lancini, M.; Serpelloni, M.; Pasinetti, S. Instrumented crutches to measure the internal forces acting on upper limbs in powered exoskeleton users. In Proceedings of 2015 6th IEEE International Workshop on Advances in Sensors and Interfaces. Gallipoli, Italy, 18-19 June, 2015. DOI. 10.1109/IWASI.2015.7184960

[3] Dijkers, M. Correlates of life satisfaction among persons with spinal cord injury. Archives of Physical Medicine and Rehabilitation, 1999, 80(8), 867-876, DOI. 10.1016/S0003-9993(99)90076-X

[4] McDonough, A.L.; Batavia, M.; Chen, F.C.; Kwon, S.; Ziai, J. The validity and reliability of the GAITRite system's measurements: a preliminary evaluation. Arch Phys Med Rehabil, 2001, 82, 419-425, DOI. 10.1053/apmr.2001.19778

[5] Tao, W; Liu, T; Zheng, R.; Feng, H. Gait analysis using wearable sensors. Sensors 2012, 12, 2255-2283, DOI. $10.3390 / \mathrm{s} 120202255$

[6] Jha, C.K.; Agarwal, S.; Chakraborty, A.L., Shirpurkar, C. An FBG-based sensing glove to measure dynamic finger flexure with an angular resolution of $0.1^{\mathrm{O}}$ up to speeds of $80^{\mathrm{O}} / \mathrm{s}$. Journal of Lightwave Technology. 2019 (early access). DOI 0.1109/JLT.2019.2919496
[7] Tsuda, N.; Hayashi, A.; Tounai, M.; Akutagawa, S. Visualization System of Crutch Walking based on Internal Sensors. In Proceedings of 2010 IEEE/ASME International Conference on Advanced Intelligence Mechatronics. MontrÃ@)al, Canada, 6-9 July, 2010.

[8] Culmer, P.R.; Brooks, P.C.; Strauss, D.N.; Ross, D.H.; Levesley, M.C.; O'Connor, R.J.; Bhakta, B.B. An instrumented walking aid to assess and retrain gait. IEEE/ASME Transactions on Mechatronics, 2014, 19(1), 141148, DOI. 10.1109/TMECH.2012.2223227

[9] Sardini, E.; Serpelloni, M.; Lancini, M. Wireless Instrumented Crutches for Force and Movement Measurements for Gait Monitoring. IEEE Transactions on Instrumentation and Measurement 2015, 64(12), 3369-3379, DOI. 10.1109/TIM.2015.2465751

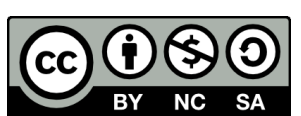

(C) 2019 by the authors. Submitted for possible open access publication under the terms and conditions of the Creative Commons Attribution CC BY-NC-SA 4.0 license (https://creativecommons.org/licenses/by-ncsa/4.0/deed.es). 